\title{
On the Linearity of Semantic Change: Investigating Meaning Variation via Dynamic Graph Models
}

\author{
Steffen Eger \\ Ubiquitous Knowledge Processing Lab \\ Department of Computer Science \\ Technische Universität Darmstadt
}

\author{
Alexander Mehler \\ Text Technology Lab \\ Department of Computer Science \\ Goethe-Universität Frankfurt am Main
}

\begin{abstract}
We consider two graph models of semantic change. The first is a time-series model that relates embedding vectors from one time period to embedding vectors of previous time periods. In the second, we construct one graph for each word: nodes in this graph correspond to time points and edge weights to the similarity of the word's meaning across two time points. We apply our two models to corpora across three different languages. We find that semantic change is linear in two senses. Firstly, today's embedding vectors (= meaning) of words can be derived as linear combinations of embedding vectors of their neighbors in previous time periods. Secondly, self-similarity of words decays linearly in time. We consider both findings as new laws/hypotheses of semantic change.
\end{abstract}

\section{Introduction}

Meaning is not uniform, neither across space, nor across time. Across space, different languages tend to exhibit different polysemous associations for corresponding terms (Eger et al., 2015; Kulkarni et al., 2015b). Across time, several wellknown examples of meaning change in English have been documented. For example, the word gay's meaning has shifted, during the 1970s, from an adjectival meaning of cheerful at the beginning of the $20^{\text {th }}$ century to its present meaning of homosexual (Kulkarni et al., 2015a). Similarly, technological progress has led to semantic broadening of terms such as transmission, mouse, or apple.
In this work, we consider two graph models of semantic change. Our first model is a $d y$ namic model in that the underlying paradigm is a (time-)series of graphs. Each node in the series of graphs corresponds to one word, associated with which is a semantic embedding vector. We then ask how the embedding vectors in one time period (graph) can be predicted from the embedding vectors of neighbor words in previous time periods. In particular, we postulate that there is a linear functional relationship that couples a word's today's meaning with its neighbor's meanings in the past. When estimating the coefficients of this model, we find that the linear form appears indeed very plausible. This functional form then allows us to address further questions, such as negative relationships between words - which indicate semantic differentiation over time - as well as projections into the future. We call our second graph model time-indexed self-similarity graphs. In these graphs, each node corresponds to a time point and the link between two time points indicates the semantic similarity of a specific word across the two time points under consideration. The analysis of these graphs reveals that most words obey a law of linear semantic 'decay': semantic self-similarity decreases linearly over time.

In our work, we capture semantics by means of word embeddings derived from context-predicting neural network architectures, which have become the state-of-the-art in distributional semantics modeling (Baroni et al., 2014). Our approach and results are partly independent of this representation, however, in that we take a structuralist approach: we derive new, 'second-order embeddings' by modeling the meaning of words by 
means of their semantic similarity relations to all other words in the vocabulary (de Saussure, 1916; Rieger, 2003). Thus, future research may in principle substitute the deep-learning architectures for semantics considered here by any other method capable of producing semantic similarity values between lexical units.

This work is structured as follows. In $\S 2$, we discuss related work. In $\S 3.1$ and 3.2, respectively, we formally introduce the two graph models outlined. In $\S 4$, we detail our experiments and in $\S 5$, we conclude.

\section{Related work}

Broadly speaking, one can distinguish two recent NLP approaches to meaning change analysis. On the one hand, coarse-grained trend analyses compare the semantics of a word in one time period with the meaning of the word in the preceding time period (Jatowt and Duh, 2014; Kulkarni et al., 2015a). Such coarse-grained models, by themselves, do not specify in which respects a word has changed (e.g., semantic broadening or narrowing), but just aim at capturing whether meaning change has occurred. In contrast, more fine-grained analyses typically senselabel word occurrences in corpora and then investigate changes in the corresponding meaning distributions (Rohrdantz et al., 2011; Mitra et al., 2014; Plitz et al., 2015; Zhang et al., 2015). Sense-labeling may be achieved by clustering of the context vectors of words (Huang et al., 2012; Chen et al., 2014; Neelakantan et al., 2014) or by applying LDA-based techniques where word contexts take the roles of documents and word senses take the roles of topics (Rohrdantz et al., 2011; Lau et al., 2012). Finally, there are studies that test particular meaning change hypotheses such as whether similar words tend to diverge in meaning over time (according to the 'law of differentiation') (Xu and Kemp, 2015) and papers that intend to detect corresponding terms across time (words with similar meanings/roles in two time periods but potentially different lexical forms) (Zhang et al., 2015).

\section{Graph models}

Let $V=\left\{w_{1}, \ldots, w_{|V|}\right\}$ be the common vocabulary (intersection) of all words in all time periods $t \in \mathcal{T}$. Here, $\mathcal{T}$ is a set of time indices. Denote an embedding of a word $w_{i}$ at time period $t$ as $\mathbf{w}_{i}(t) \in \mathbb{R}^{d}$. Since embeddings $\mathbf{w}_{i}(s), \mathbf{w}_{i}(t)$ for two different time periods $s, t$ are generally not comparable, as they may lie in different coordinate systems, we consider the vectors $\tilde{\mathbf{w}}_{i}(t)=$

$$
\left(\operatorname{sim}\left(\mathbf{w}_{i}(t), \mathbf{w}_{1}(t)\right), \ldots, \operatorname{sim}\left(\mathbf{w}_{i}(t), \mathbf{w}_{|V|}(t)\right)\right),
$$

each of which lies in $\mathbb{R}^{|V|}$ and where sim is a similarity function such as the cosine. We note that our structuralist definition of $\tilde{\mathbf{w}}_{i}(t)$ is not unproblematic, since the vectors $\mathbf{w}_{1}(t), \ldots, \mathbf{w}_{|V|}(t)$ tend to be different across $t$, by our very postulate, so that there is non-identity of these 'reference points' over time. However, as we may assume that the meanings of at least a few words are stable over time, we strongly expect the vectors $\tilde{\mathbf{w}}_{i}(t)$ to be suitable for our task of analysis of meaning changes. ${ }^{1}$ For the remainder of this work, for convenience, we do not distinguish, in terms of notation, between $\mathbf{w}_{i}(t)$ and $\tilde{\mathbf{w}}_{i}(t)$.

\subsection{A linear model of semantic change}

We postulate, and subsequently test, the following model of meaning dynamics which describes meaning change over time for words $w_{i}$ :

$$
\mathbf{w}_{i}(t)=\sum_{n=1}^{p} \sum_{w_{j} \in V \cap N\left(w_{i}\right)} \alpha_{w_{j}}^{n} \mathbf{w}_{j}(t-n)
$$

where $\alpha_{w_{j}}^{n} \in \mathbb{R}$, for $n=1, \ldots, p$, are coefficients of meaning vectors $\mathbf{w}_{j}(t-n)$ and $p \geq 1$ is the order of the model. The set $N\left(w_{i}\right) \subseteq V$ denotes a set of 'neighbors' of word $w_{i}{ }^{2}$ This model says that the meaning of a word $w_{i}$ at some time $t$ is determined by reference to the meanings of its 'neighbors' in previous time periods, and that the underlying functional relationship is linear.

We remark that the model described by Eq. (2) is a time-series model, and, in particular, a vector-autoregressive (VAR) model with special

\footnotetext{
${ }^{1}$ An alternative to our second-order embeddings is to project vectors from different time periods in a common space (Mikolov et al., 2013a; Faruqui and Dyer, 2014), which requires to find corresponding terms across time. Further, one could also consider a 'core' vocabulary of semantically stable words, e.g., in the spirit of Swadesh (1952), instead of using all vocabulary words as reference.

${ }^{2}$ We also constrain the vectors $\mathbf{w}_{i}(t)$, for all $w_{i} \in V$, to contain non-zero entries only for words in $N\left(w_{i}\right)$.
} 
structure. The model may also be seen in the socio-economic context of so-called "opinion dynamic models" (Golub and Jackson, 2010; Acemoglu and Ozdaglar, 2011; Eger, 2016). There it is assumed that agents are situated in network structures and continuously update their opinions/beliefs/actions according to their ties with other agents. Model (2) substitutes multi-dimensional embedding vectors for onedimensional opinions.

\subsection{Time-indexed self-similarity graphs}

We track meaning change by considering a fully connected graph $G(w)$ for each word $w$ in $V$. The nodes of $G(w)$ are the time indices $\mathcal{T}$, and there is an undirected link between any two $s, t \in \mathcal{T}$ whose weight is given by $\operatorname{sim}(\mathbf{w}(s), \mathbf{w}(t))$. We call the graphs $G(w)$ time-indexed self-similarity (TISS) graphs because they indicate the (semantic) similarity of a given word with itself across different time periods. Particular interest may lie in weak links in these graphs as they indicate low similarity between two different time periods, i.e., semantic change across time.

\section{Experiments}

Data As corpus for English, we use the Corpus of Historical American (COHA). ${ }^{3}$ This covers texts from the time period 1810 to 2000 . We extract two slices: the years 1900-2000 and 1810-2000. For both slices, each time period $t$ is one decade, e.g., $\mathcal{T}=\{1810,1820,1830, \ldots\} .{ }^{4}$ For each slice, we only keep words associated to the word classes nouns, adjectives, and verbs. For computational and estimation purposes, we also only consider words that occur at least 100 times in each time period. To induce word embeddings $\mathbf{w} \in \mathbb{R}^{d}$ for each word $w \in V$, we use word2vec (Mikolov et al., 2013b) with default parametrizations. We do so for each time period $t \in \mathcal{T}$ independently. We then use these embeddings to derive the new embeddings as in Eq. (1). Throughout, we use cosine similarity as sim measure. For German, we consider a proprietary dataset of the German newspaper $S Z^{5}$ for which $\mathcal{T}=\{1994,1995, \ldots, 2003\}$.

\footnotetext{
${ }^{3}$ http://corpus.byu.edu/coha/.

${ }^{4}$ Each time period contains texts that were written in that decade.

${ }^{5}$ http://www.sueddeutsche.de/
}

We lemmatize and POS tag the data and likewise only consider nouns, verbs and adjectives, making the same frequency constraints as in English. Finally, we use the PL (Migne, 1855) as data set for Latin. Here, $\mathcal{T}=\{300,400, \ldots, 1300\}$. We use the same preprocessing, frequency, and word class constraints as for English and German.

Throughout, our datasets are well-balanced in terms of size. For example, the English COHA datasets contain about 24M-30M tokens for each decade from 1900 to 2000, where the decades 1990 and 2000 contain slighly more data than the earlier decades. The pre-1900 decades contain 18$24 \mathrm{M}$ tokens, with only the decades 1810 and 1820 containing very little data $(1 \mathrm{M}$ and $7 \mathrm{M}$ tokens, respectively). The corpora are also balanced by genre.

\subsection{TISS graphs}

We start with investigating the TISS graphs. Let $D_{t_{0}}$ represent how semantically similar a word is across two time periods, on average, when the distance between time periods is $t_{0}: D_{t_{0}}=$ $\frac{1}{C} \sum_{w \in V} \sum_{|s-t|=t_{0}} \operatorname{sim}(\mathbf{w}(s), \mathbf{w}(t))$, where $C=$ $|V| \cdot\left|\left\{(s, t)|| s-t \mid=t_{0}\right\}\right|$ is a normalizer. Figure 1 plots the values $D_{t_{0}}$ for the time slice from 1810 to 2000, for the English data. We notice a clear trend: self-similarity of a word tends to (almost perfectly) linearly decrease with time distance. In

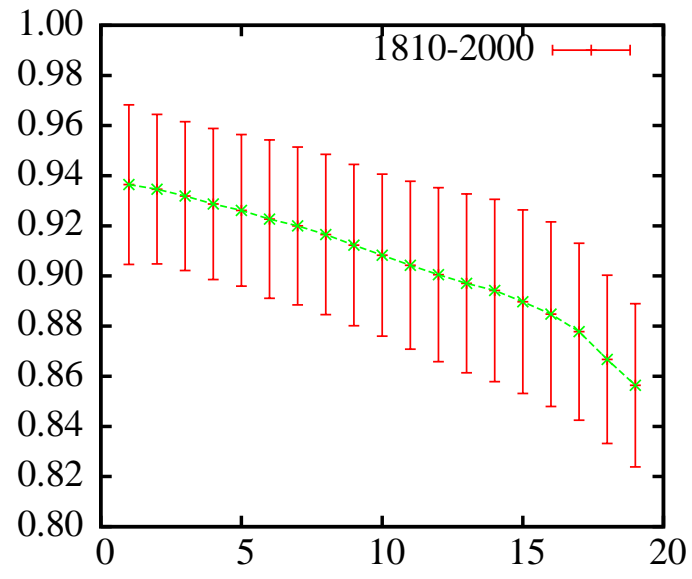

Figure 1: $D_{t_{0}}$ (y-axis) as a function of $t_{0}(x$-axis), values of $D_{t_{0}}$ (in green) and error-bars.

fact, Table 1 below indicates that this trend holds across all our corpora, i.e., for different time scales and different languages: the linear 'decay' model 
fits the $D_{t_{0}}$ curves very well, with adjusted $R^{2}$ values substantially above $90 \%$ and consistently and significantly negative coefficients. We believe that this finding may be considered a new statistical law of semantic change.

\begin{tabular}{|llllll|}
\hline Corpus Lang. & $\begin{array}{l}\text { Time } \\
\text { interval }\end{array}$ & Years & Coeff. & $R^{2}$ \\
\hline COHA English & Decade & $1900-2000$ & -0.425 & 98.63 \\
& & $1810-2000$ & -0.405 & 96.03 \\
SZ & German & Year & $1994-2003$ & -0.678 & 98.64 \\
PL & Latin & Century & $400-1300$ & -0.228 & 92.28 \\
\hline
\end{tabular}

Table 1: Coefficients (\%) in regression of $D_{t_{0}}$ on $t_{0}$, and adjusted $R^{2}$ values (\%).

The values $D_{t_{0}}$ as a function of $t_{0}$ are averages over all words. Thus, it might be possible that the average word's meaning decays linearly in time, while the semantic behavior, over time, of a large fraction of words follows different trends. To investigate this, we consider the distribution of $D_{t_{0}}(w)=\frac{1}{C^{\prime}} \sum_{|s-t|=t_{0}} \operatorname{sim}(\mathbf{w}(s), \mathbf{w}(t))$ over fixed words $w$. Here $C^{\prime}=\left|\left\{(s, t)|| s-t \mid=t_{0}\right\}\right|$. We consider the regression models

$$
D_{t_{0}}(w)=\alpha \cdot t_{0}+\text { const. }
$$

for each word $w$ independently and assess the distribution of coefficients $\alpha$ as well as the goodnessof-fit values. Figure 2 shows - exemplarily for the English 1900-2000 COHA data - that the coefficients $\alpha$ are negative for almost all words. In fact, the distribution is left-skewed with a mean of around $-0.4 \%$. Moreover, the linear model is always a good to very good fit of the data in that $R^{2}$ values are centered around $85 \%$ and rarely fall below $75 \%$. We find similar patterns for all other datasets considered here. This shows that not only the average word's meaning decays linearly, but almost all words' (whose frequency mass exceeds a particular threshold) semantics behaves this way.

Next, we use our TISS graphs for the task of finding words that have undergone meaning change. To this end, we sort the graphs $G(w)$ by the ratios $R_{G(w)}=\frac{\text { maxlink }}{\text { minlink }}$, where maxlink denotes maximal weight of a link in graph $G(w)$ and minlink is the minimal weight of a link in graph $G(w)$. We note that weak links may indicate semantic change, but the stated ratio requires that 'weakness' is seen relative to the strongest semantic links in the TISS graphs. Table 2 presents
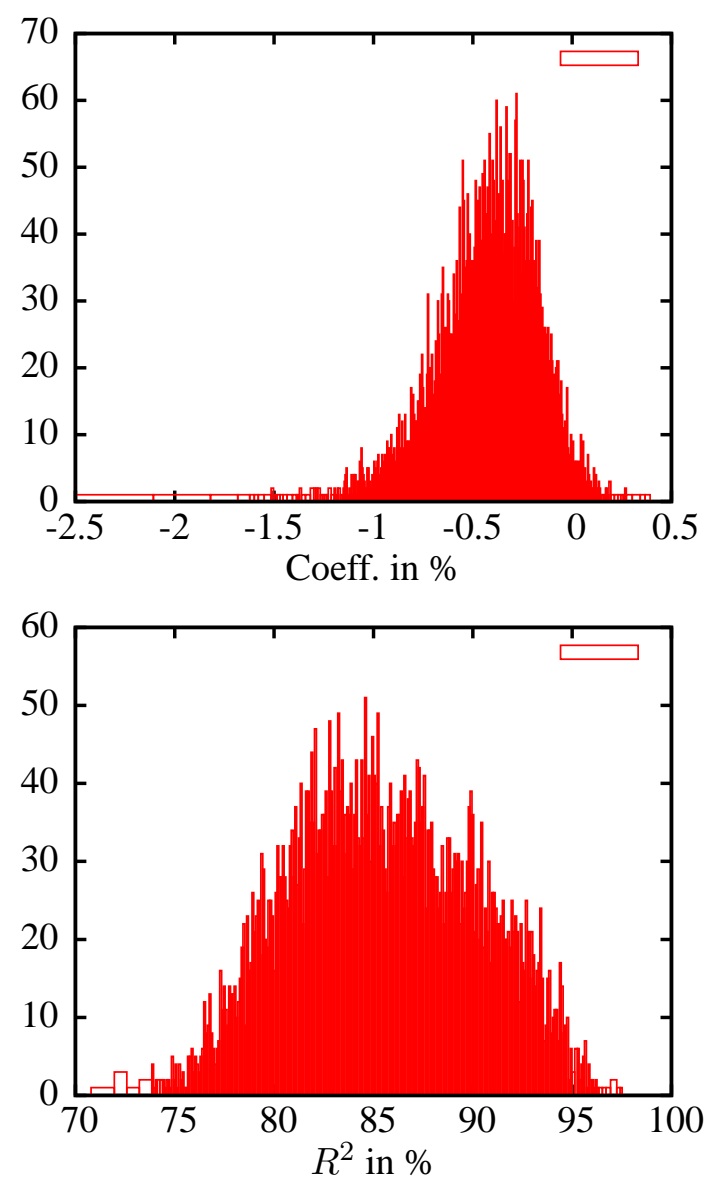

Figure 2: Distribution of Coefficients $\alpha$ (top) and $R^{2}$ values (bottom) in regression of values $D_{t_{0}}(w)$ on $t_{0}$. The plots are histograms: $y$-axes are frequencies.

selected words that have highest values $R_{G(w)} \cdot{ }^{6}$ We omit a fine-grained semantic change analysis,

bush (1), web (2), alan (3), implement (4)
jeff (5), gay (6), program (7), film (8),
focus (9), terrific (16), axis (36)

Table 2: Selected words with highest values $R_{G(w)}$ in COHA for the time period 1900-2000. In brackets are the ranks of words, i.e., bush has the highest value $R_{G(w)}$, web the 2 nd highest, etc.

which could be conducted via the methods outlined in $\S 2$, but notice a few cases. 'Terrific' has a large semantic discrepancy between the 1900s and

\footnotetext{
${ }^{6}$ The top ten words with the lowest values $R_{G(w)}$ are one, write, have, who, come, only, even, know, hat, fact.
} 
the 1970s, when the word probably (had) changed from a negative to a more positive meaning. The largest discrepancy for 'web' is between the 1940s and the 2000s, when it probably came to be massively used in the context of the Internet. The high $R_{G(w)}$ value for $w=$ 'axis' derives from comparing its use in the 1900s with its use in the 1940s, when it probably came to be used in the context of Nazi Germany and its allies. We notice that the presented method can account for gradual, accumulating change, which is not possible for models that compare two succeeding time points such as the model of Kulkarni et al. (2015a).

\subsection{Meaning dynamics network models}

Finally, we estimate meaning dynamics models as in Eq. (2), i.e., we estimate the coefficients $\alpha_{w_{j}}^{n}$ from our data sources. We let the neighbors $N(w)$ of a word $w$ as in Eq. (2) be the union (w.r.t. $t$ ) over sets $N_{t}(w ; n)$ denoting the $n \geq 1$ semantically most similar words (estimated by cosine similarity on the original word2vec vectors) of word $w$ in time period $t \in \mathcal{T}^{7}$ In Table 3, we indicate two measures: adjusted $R^{2}$, which indicates the goodness-of-fit of a model, and prediction error. By prediction error, we measure the average Euclidean distance between the true semantic vector of a word in the final time period $t_{N}$ vs. the predicted semantic vector, via the linear model in Eq. (2), estimated on the data excluding the final period. The indicated prediction error is the average over all words. We note the following: $R^{2}$ values are high (typically above 95\%), indicating that the linear semantic change model we have suggested fits the data well. Moreover, $R^{2}$ values slightly increase between order $p=1$ and $p=2$; however, for prediction error this trend is reversed. ${ }^{8}$ We also include a strong baseline that claims that word meanings do not change in the final period $t_{N}$ but are the same as in $t_{N-1}$. We note that the order $p=1$ model consistently improves upon this baseline, by as much as $18 \%$, depending upon parameter settings.

Negative relationships Another very interest-

\footnotetext{
${ }^{7}$ We exclude word $w$ from $N_{t}(w ; n)$. We found that including $w$ did not improve performance results.

${ }^{8}$ We experimented with orders $p \geq 3$, but found them to be inadequate. Typically, coefficients for lagged-3 variables are either zero or model predictions are way off, possibly indicating multi-collinearity.
}

\begin{tabular}{ccccc}
$n$ & $p$ & Adjusted- $R^{2}$ & Pred. Error & Baseline \\
\hline 5 & 1 & $95.68 \pm 2.80$ & $0.402 \pm .234$ & $0.447 \pm .232$ \\
& 2 & $96.13 \pm 1.83$ & $0.549 \pm .333$ & \\
10 & 1 & $95.24 \pm 2.78$ & $0.378 \pm .169$ & $0.445 \pm .187$ \\
& 2 & $95.75 \pm 2.67$ & $0.515 \pm .247$ & \\
20 & 1 & $94.72 \pm 2.85$ & $0.362 \pm .127$ & $0.442 \pm .156$ \\
& 2 & $95.27 \pm 2.74$ & $0.493 \pm .190$ &
\end{tabular}

Table 3: English data, 1900-2000. $R^{2}$ and prediction error in \%.

ing aspect of the model in Eq. (2) is that it allows for detecting words $w_{j}$ whose embeddings have negative coefficients $\alpha_{w_{j}}$ for a target word $w_{i}$ (we consider $p=1$ in the remainder). Such negative coefficients may be seen as instantiations of the 'law of differentiation': the two words' meanings move, over time, in opposite directions in semantic space. We find significantly negative relationships between the following words, among others: summit $\leftrightarrow$ foot, boy $\leftrightarrow$ woman, vow $\leftrightarrow$ belief, negro $\leftrightarrow$ black. Instead of a detailed analysis, we mention that the Wikipedia entry for the last pair indicates that the meanings of 'negro' and 'black' switched roles between the early and late $20^{\text {th }}$ century. While 'negro' was once the "neutral" term for the colored population in the US, it acquired negative connotations after the 1960s; and vice versa for 'black'.

\section{Concluding remarks}

We suggested two novel models of semantic change. First, TISS graphs allow for detecting gradual, non-consecutive meaning change. They enable to detect statistical trends of a possibly general nature. Second, our time-series models allow for investigating negative trends in meaning change ('law of differentiation') as well as forecasting into the future, which we leave for future work. Both models hint at a linear behavior of semantic change, which deserves further investigation. We note that this linearity concerns the core vocabulary of languages (in our case, words that occurred at least 100 times in each time period), and, in the case of TISS graphs, is an average result; particular words may have drastic, non-linear meaning changes across time (e.g., proper names referring to entirely different entities). However, our analysis also finds that most core words' meanings decay linearly in time. 


\section{References}

Daron Acemoglu and Asuman Ozdaglar. 2011. Opinion dynamics and learning in social networks. $D y$ namic Games and Applications, 1(1):3-49.

Marco Baroni, Georgiana Dinu, and Germán Kruszewski. 2014. Don't count, predict! a systematic comparison of context-counting vs. context-predicting semantic vectors. In Proceedings of the 52nd Annual Meeting of the Association for Computational Linguistics (Volume 1: Long Papers), pages 238-247, Baltimore, Maryland, June. Association for Computational Linguistics.

Xinxiong Chen, Zhiyuan Liu, and Maosong Sun. 2014. A unified model for word sense representation and disambiguation. In Proceedings of the 2014 Conference on Empirical Methods in Natural Language Processing, EMNLP 2014, October 25 29, 2014, Doha, Qatar, A meeting of SIGDAT, a Special Interest Group of the ACL, pages 1025-1035.

Ferdinand de Saussure. 1916. Cours de linguistique générale. Payot, Lausanne/Paris.

Steffen Eger, Niko Schenk, and Alexander Mehler. 2015. Towards semantic language classification: Inducing and clustering semantic association networks from europarl. In Proceedings of the Fourth Joint Conference on Lexical and Computational Semantics, pages 127-136, Denver, Colorado, June. Association for Computational Linguistics.

Steffen Eger. 2016. Opinion dynamics and wisdom under out-group discrimination. Mathematical Social Sciences, 80(C):97-107.

Manaal Faruqui and Chris Dyer. 2014. Improving vector space word representations using multilingual correlation. In Proceedings of EACL.

Benjamin Golub and Matthew O. Jackson. 2010. Nave learning in social networks and the wisdom of crowds. American Economic Journal: Microeconomics, 2(1):112-49, February.

Eric H. Huang, Richard Socher, Christopher D. Manning, and Andrew Y. Ng. 2012. Improving word representations via global context and multiple word prototypes. In Proceedings of the 50th Annual Meeting of the Association for Computational Linguistics: Long Papers - Volume 1, ACL '12, pages 873-882, Stroudsburg, PA, USA. Association for Computational Linguistics.

Adam Jatowt and Kevin Duh. 2014. A framework for analyzing semantic change of words across time. In Proceedings of the Joint JCDL/TPDL Digital Libraries Conference.

Vivek Kulkarni, Rami Al-Rfou, Bryan Perozzi, and Steven Skiena. 2015a. Statistically significant detection of linguistic change. In Proceedings of the 24th International Conference on World Wide Web, WWW'15, pages 625-635, Republic and Canton of Geneva, Switzerland. International World Wide Web Conferences Steering Committee.

Vivek Kulkarni, Bryan Perozzi, and Steven Skiena. 2015b. Freshman or fresher? quantifying the geographic variation of internet language. CoRR, abs/1510.06786.

Jey Han Lau, Paul Cook, Diana McCarthy, David Newman, and Timothy Baldwin. 2012. Word sense induction for novel sense detection. In Proceedings of the 13th Conference of the European Chapter of the Association for Computational Linguistics, EACL '12, pages 591-601, Stroudsburg, PA, USA. Association for Computational Linguistics.

Jacques-Paul Migne, editor. 1855. Patrologiae cursus completus: Series latina. 1-221. ChadwyckHealey, Cambridge.

Tomas Mikolov, Quoc V. Le, and Ilya Sutskever. 2013a. Exploiting similarities among languages for machine translation. CoRR, abs/1309.4168.

Tomas Mikolov, Ilya Sutskever, Kai Chen, Greg S Corrado, and Jeff Dean. 2013b. Distributed representations of words and phrases and their compositionality. In C.J.C. Burges, L. Bottou, M. Welling, Z. Ghahramani, and K.Q. Weinberger, editors, $A d$ vances in Neural Information Processing Systems 26, pages 3111-3119. Curran Associates, Inc.

Sunny Mitra, Ritwik Mitra, Martin Riedl, Chris Biemann, Animesh Mukherjee, and Pawan Goyal. 2014. That's sick dude!: Automatic identification of word sense change across different timescales. In Proceedings of the 52nd Annual Meeting of the Association for Computational Linguistics, ACL 2014, June 22-27, 2014, Baltimore, MD, USA, Volume 1: Long Papers, pages 1020-1029.

Arvind Neelakantan, Jeevan Shankar, Alexandre Passos, and Andrew McCallum. 2014. Efficient nonparametric estimation of multiple embeddings per word in vector space. In Proceedings of the 2014 Conference on Empirical Methods in Natural Language Processing, EMNLP 2014, October 25-29, 2014, Doha, Qatar, A meeting of SIGDAT, a Special Interest Group of the ACL, pages 1059-1069.

Christian Plitz, Thomas Bartz, Katharina Morik, and Angelika Strrer. 2015. Investigation of word senses over time using linguistic corpora. In Pavel $\mathrm{Krl}$ and Vclav Matouek, editors, Text, Speech, and Dialogue, volume 9302 of Lecture Notes in Computer Science, pages 191-198. Springer International Publishing.

Burghard B. Rieger. 2003. Semiotic cognitive information processing: Learning to understand discourse. A systemic model of meaning constitution. 
In R. Kühn, R. Menzel, W. Menzel, U. Ratsch, M. M. Richter, and I. O. Stamatescu, editors, Adaptivity and Learning. An Interdisciplinary Debate, pages 347-403. Springer, Berlin.

Christian Rohrdantz, Annette Hautli, Thomas Mayer, Miriam Butt, Daniel A. Keim, and Frans Plank. 2011. Towards tracking semantic change by visual analytics. In The 49th Annual Meeting of the Association for Computational Linguistics: Human Language Technologies, Proceedings of the Conference, 19-24 June, 2011, Portland, Oregon, USA - Short Papers, pages 305-310.

Morris Swadesh. 1952. Lexico-statistic dating of prehistoric ethnic contacts. Proceedings of the American Philosophical Society, 96(4):452463.

Y. Xu and C. Kemp. 2015. A computational evaluation of two laws of semantic change. In Proceedings of the 37th Annual Conference of the Cognitive Science Society.

Yating Zhang, Adam Jatowt, Sourav S. Bhowmick, and Katsumi Tanaka. 2015. Omnia mutantur, nihil interit: Connecting past with present by finding corresponding terms across time. In Proceedings of the 53rd Annual Meeting of the Association for Computational Linguistics and the 7th International Joint Conference on Natural Language Processing of the Asian Federation of Natural Language Processing, ACL 2015, July 26-31, 2015, Beijing, China, Volume 1: Long Papers, pages 645-655. 